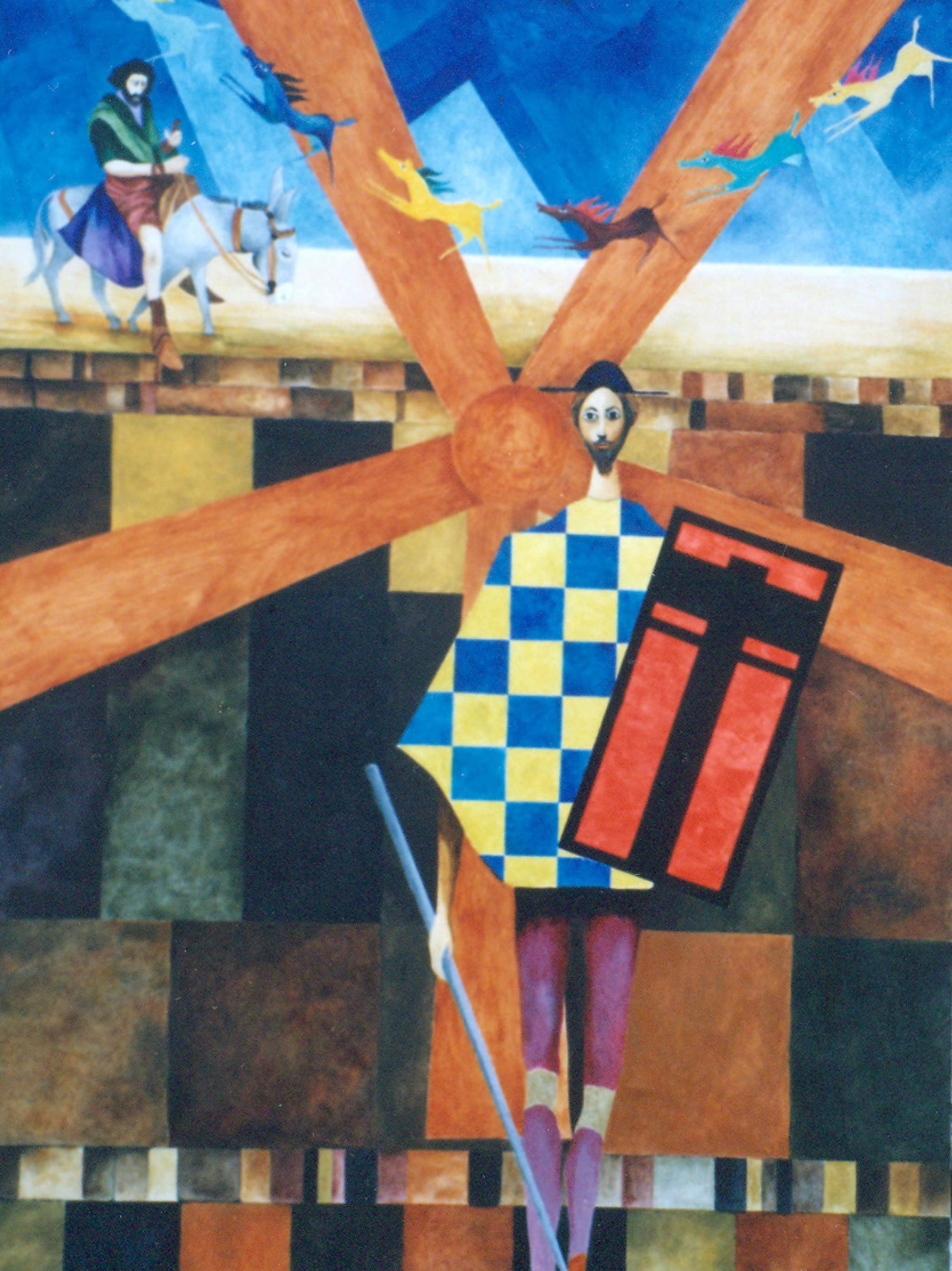




\section{Crisis laboral en la región metropolitana Guadalajara}

José María Parra Ruíz*, Emilia Gámez Frías**

RESUMEN. Con base en la información estadística disponible, se observa que desde 2014, la población de la región metropolitana Guadalajara (RMG) ${ }^{1}$ enfrenta la mayor crisis laboral del presente siglo. Al cabo de un año, poco más de 70 mil trabajadores, perdieron su empleo y 5 mil más pasaron a engrosar las filas del desempleo. Asimismo, se observa que un mayor número de trabajadores percibió un ingreso inferior al que se requiere para adquirir la canasta básica recomendable. Por lo tanto se considera que la pérdida de empleo, así como, los bajos niveles de ingreso que perciben los trabajadores de este territorio metropolitano, producto de la intensificación en el ritmo de acumulación de capital y los cambios laborales y fiscales, configuran una crisis laboral superior a la ocurrida en 2009 (año de crisis económica nacional, regional e internacional).

Palabras Clave: Crisis laboral; Nivel de empleo; Nivel salarial.

ABSTRACT. Based on the statistical information available, it appears that since 2014, the population of the Guadalajara metropolitan area (RMG) faces the greatest employment crisis of this century. After a year, just over 70 thousand workers lost their jobs and 5000 more were added to the unemployment lines. It also shows that a greater number of workers saw an income below that required to purchase the recommended basic food basket. Therefore we believe that the loss of employment as well as low levels of income earned by workers in this metropolitan area, due to the escalation in the rate of accumulation of capital and labor and tax changes, form a labor crisis than the one in 2009 (year of national, regional and international economic crisis).

Keywords: Job crisis; Level of employment; salary level.

\section{Introducción}

En el diagnóstico laboral a escala nacional y de las principales metrópolis de México, se asevera que la población ocupada enfrenta un mayor deterioro, en distintas formas y grados, en sus condiciones laborales. En los diferentes estudios, se ofrece como evidencia que dicho deterioro se observa en los bajos niveles de empleo (principalmente formal), así como, en la inestabilidad en el empleo, los bajos ingresos, falta de seguridad social y de prestaciones laborales, tanto en el sector formal como informal. Todo ello, como consecuencia de los magros resultados que expresa la economía nacional (conducida, aseveran, desde 1983, bajo la estrategia económica neoliberal) y la falta de inversión pública y privada que permitan generar empleos suficientes, decentes o dignos (Calva y Salazar, 2012; Aguilar y Escamilla, 2000; Aguiar, 2014).

Los estudios sobre el deterioro laboral ${ }^{2}$ en México, son de la mayor importancia (Sotelo, 2012) y constituyen un objeto de estudio para diversas y disímbolas perspectivas teórico-conceptuales-metodológicas, que ofrecen numerosas valoraciones sobre dicho objeto (Hualde y Guadarrama, 2012; Antunes, 2012; Alves, 2007; Gómez, 2007). Sin embargo, existe un cierto consenso respecto a que, en los últimos años, aumentó el deterioro laboral a escala nacional. El diagnóstico se ofrece, principalmente, para el mercado de trabajo, así como para el uso de la fuerza de trabajo.

Recibido en mayo 2015/ acepatado y versión final julio 2015

* Dr. En Ciencias Sociales por el COLEF,Profesor-Investigador, Departamento de Estudios Regionales-INESER, UDG. jparra@cucea.udg.mx. Línea de Investigación: Configuración socio-espacial de la fuerza de trabajo transnacional mexicana. Especialidad en Estudios Regionales.

** Maestra en Economía y Negocios por la Universidad de Guadalajara. Profesor-Investigador, Departamento de Estudios Regionales-INESER, Universidad de Guadalajara, emigamez@cucea.udg.mx, Línea de Investigación: Configuración socio-espacial de la fuerza de trabajo transnacional mexicana. Especialidad en Estudios Regionales.

1. La RMG la configuran los municipios de Guadalajara, Zapopan, Tlaquepaque, Tonalá, Tlajomulco de Zúñiga y El Salto, Jalisco. Para una explicación amplia véase (Parra y Gámez, 2006).

2. Sin embargo, Rojas y Salas (sf), consideran que a pesar de que en México existe una diversidad de estudios sobre la calidad de los empleos resultan insuficientes los correspondientes a la precariedad laboral. 
Destacan estudios de los mercados de fuerza de trabajo metropolitanos tales como el de la RMG. En esta vertiente de estudios se compara la precariedad que registran Guadalajara, Ciudad de México y Monterrey. En los trabajos de García (2010, 2009); Rubio, (2010) y Gaxiola, (2012), se sostiene que la precariedad laboral en la metrópoli de Guadalajara se encuentra fuertemente arraigada en la estructura ocupacional al igual que en la ciudad de México. Sin embargo, se concluye que ambas metrópolis se encuentran por arriba del nivel de precariedad que registra Monterrey (Gaxiola, 2013). Esta valoración, se sustenta en el análisis de una serie de indicadores: integrales, conglomerados y componentes principales, tasas de crecimiento y de participación porcentual respecto a la estructura y dinámica del mercado de trabajo. Las dimensiones analizadas, principalmente para un año de referencia y en menor número para series de tiempo, son: comportamiento ocupacional, contratos de trabajo, prestaciones sociales, incorporación a las instituciones de salud, niveles salariales, jornada de trabajo, niveles de sindicalización, entre otros (García, 2009; Rubio, 2010).

Gráfico 1. RMG: tasa de crecimiento de indicadores económicos, 2008-2014

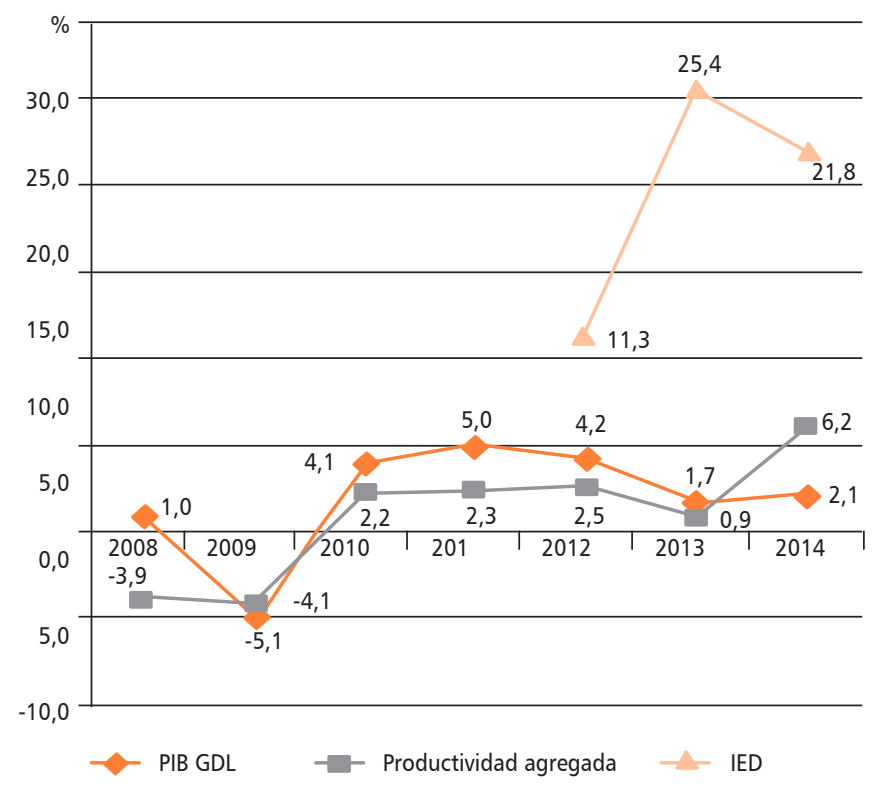

Fuente: PIB: Indicadores Regionales de Actividad Banamex, Estimaciones de PIB elaboradas por el Departamento de Estudios Económicos.

Productividad: Cálculo propio con base en INEGI, 2015

IED: Cálculo propio con base en Secretaría de Economía, 2015
En esta trayectoria de estudios, se inscribe el presente trabajo. El argumento central es que la población de la RMG, experimenta, en 2014, un mayor deterioro en su nivel de ocupación y salarial en un contexto de exiguo y volátil de crecimiento económico, de aumento en la productividad agregada y en la inversión extranjera directa (Gráfico 1).

\section{Metodología}

Con el fin de ofrecer evidencia sobre tan adversa situación laboral en la RMG, se analiza el dinamismo que registra el nivel de empleo (en términos generales y, por rama de actividad económica), así como el nivel salarial (su comportamiento general y por rama de actividad económica) por cada uno de los cuatro segmentos de trabajadores que configuran la estructura ocupacional.

Cabe señalar que el análisis de la información estadística: Encuesta Nacional del Empleo y la Ocupación (ENOE, segundo trimestre de cada año del periodo ${ }^{3}$ ), se fundamenta en la perspectiva teórica conceptual de la economía política y sociología del trabajo y en dos consideraciones institucionales (normativas: cambios fiscales y, cambios en la Ley Federal del Trabajo).

La interrogante que guía el presente trabajo es icuál es la situación que enfrentan los sujetos laborales en la RMG? Con el fin de ofrecer una respuesta a dicha interrogante, se dimensiona la crisis laboral que enfrentan los trabajadores de la RMG, en los distintos niveles y matices que ésta adquiere. Para cumplir con dicho propósito, el trabajo se divide además de esta introducción en tres apartados. En el primero, se analiza el nivel del empleo regional en términos generales, por posición en la ocupación y por rama de actividad económica. En el segundo, se analiza el nivel salarial de acuerdo a su comportamiento regional, por posición en la ocupación y por rama de actividad económica. Por último, en las consideraciones finales se enfatiza como el Estado mexicano en santa

3. Seleccionamos el segundo trimestre, de cada año, en razón de que resulta ser el que en menor medida se encuentra afectado por las fluctuaciones estacionales de la actividad económica; así como porque, en dicho trimestre, se utiliza una versión ampliada del Cuestionario ENOE. 
alianza con la élite empresarial contravienen los postulados constitucionales y de toda ética de responsabilidad social al impulsar una serie de acciones que deterioran las condiciones laborales y de vida de la población al no proporcionar empleo suficiente y decente.

\section{Discusión de resultados}

\subsection{Nivel de la Ocupación}

En 2014, la población de la Región Metropolitana Guadalajara (RMG) experimentó la mayor pérdida de empleos del presente siglo, y, también registra el mayor número de desempleados. El número de trabajadores ocupados disminuyó en poco más de 70 mil personas, de los cuales, el 72.6 por ciento fueron mujeres y el restante 27.4 por ciento son hombres. Esta pérdida supera en 49 por ciento, a la pérdida total de población ocupada en 2009 (año de crisis socioeconómica regional, nacional e internacional). En consecuencia, se configura una dinámica negativa del empleo: (-)3.9 por ciento (tasa superior en 1.2 puntos porcentuales al decremento ocupacional de 2009) ${ }^{4}$. Los primeros síntomas de dicha debacle se observan en el periodo 2011-2013, cuando la ocupación de manera consistente pierde dinamismo (Cuadro 1). Las mujeres registran una dinámica negativa superior a la de los hombres, lo cual constituye un factor explicativo sobre el comportamiento del empleo regional (Gráfico 2).

Por otra parte, 5 mil, hombres y mujeres, se sumaron a las filas del desempleo en 2014. De esta forma la tasa de
Gráfico 2. RMG: tasa de crecimiento anual de la ocupación según sexo, 2008-2014

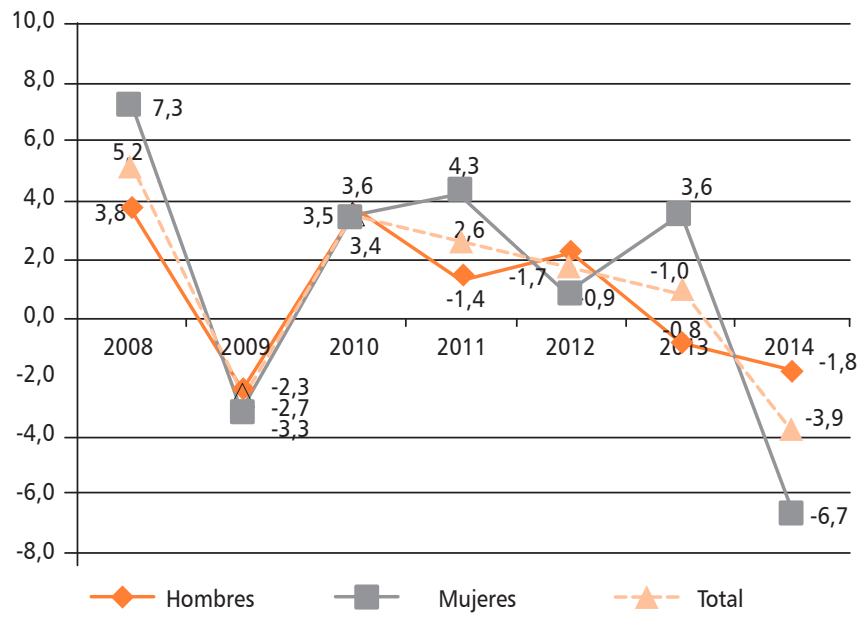

Fuente: Elaboración propia con base en INEGI, Encuesta Nacional de Ocupación y Empleo (ENOE).

desempleo se colocó en 5.4 por ciento (0.4 puntos porcentuales superior a la tasa de 2013). Este ligero incremento en la tasa de desempleo no se explica únicamente por el menor nivel de ocupación, sino principalmente, por el descenso que experimenta la población económicamente activa -3.4 por ciento ( 2.8 puntos porcentuales mayor que el descenso de la PEA en 2009) ${ }^{5}$ y, por la menor participación que registra la población en el mercado de trabajo, durante el periodo de estudio: 59.9 por ciento (Cuadro 1). Cabe señalar que desde 2009 la tasa de desempleo es superior al 5 por ciento.

En suma, el año pasado la población de la RMG experimentó la mayor debacle en su nivel de ocupación de

Cuadro 1. RMG: tasa de crecimiento y de participación de la población en la actividad económica

\begin{tabular}{|l|c|c|c|c|c|c|c|}
\hline & 2008 & 2009 & 2010 & 2011 & 2012 & 2013 \\
\hline Tasa de Crecimiento: PEA & 4.8 & -0.6 & 4.0 & 2.3 & 1.5 & 0.7 & -3.4 \\
\hline Tasa Crecimiento: Ocupación & 5.2 & -2.7 & 3.5 & 2.6 & 1.7 & 1.0 & -3.9 \\
\hline Tasa de Desempleo & 3.1 & 5.2 & 5.6 & 5.4 & 5.2 & 5.0 & 5.4 \\
\hline Tasa de Participación en el MT & 64.6 & 62.5 & 62.8 & 63.5 & 63.5 & 63.0 & 59.9 \\
\hline
\end{tabular}

Fuente: Elaboración propia con base en INEGI, Encuesta Nacional de Ocupación y Empleo (ENOE).

4. En 2008, el total de la población ocupada fue de 1 millón 725 mil 929; en 2009, 1 millón 678 mil 468; 2013: 1 millón 830 mil 384; en 2014, 1 millón 759 mil 665.
5. De acuerdo a la información agregada disponible, un factor que explica la disminución de la PEA, es que alrededor de 100 mil personas (principalmente mujeres), que en 2013, formaban parte de la PEA regional, se sumaron, al año siguiente, a la población no económicamente activa (PNEA). Se trata de hombres y mayormente de mujeres jóvenes que estudian o realizan labores en el hogar y que trabajaban por cuenta propia; así como de pensionados y jubilados. 
los tres últimos quinquenios y, también, registró el mayor número de personas desempleadas desde 2010. Con la finalidad de matizar la debacle ocupacional, el análisis se centra en la dinámica que registran los sujetos sociales con empleo en dos de sus ámbitos: por posición en la ocupación y, por su posición en la actividad económica urbana.

\subsubsection{Posición en la ocupación ${ }^{6}$}

En este apartado se analiza cada uno de los segmentos de trabajadores que configuran la estructura ocupacional en tres cuestiones: participación porcentual que tienen en el universo de la población ocupada; total de empleos perdidos; y, tasa de crecimiento anual. Ello, con el fin de establecer una aproximación a conocer y explicar la crisis laboral en diferentes niveles.

La población ocupada se estructura en cuatro segmentos de trabajadores: subordinados y remunerados ${ }^{7}$, empleadores $^{8}$, por cuenta propia ${ }^{9}$ y no remunerados ${ }^{10}$. La participación de los trabajadores subordinados y remunerados, representa casi tres cuartas partes del total de trabajadores que cuentan con una ocupación en la RMG. Además de ser la mayor del periodo de estudio (73.9 por ciento en 2014), supera en 6 puntos porcentuales la participación de la fuerza de trabajo a escala nacional y en 1.1 puntos porcentuales la participación que éstos mismos adquieren en la ciudad de México. Dicha participación constituye un buen indicador acerca de la importancia que tiene la fuerza de trabajo en la economía de la RMG, así como, en el mercado de trabajo regional toda vez que 7.4 de cada 10 trabajadores son subordinados y remunerados.

6. De acuerdo con el Instituto Nacional de Estadística Geografía e Informática (INEGI), la posición en la ocupación es la "situación que distingue a la población ocupada según la relación de propiedad con el negocio, empresa o establecimiento, y las exigencias del mismo para que contrate o no a trabajadores a cambio de un pago o sólo reciba ayuda de ocupados sin pago".

7. "Trabajador subordinado y remunerado, es la persona que trabaja (con o sin pago) para una unidad económica en la que depende de un patrón o un representante de él o de un trabajador por cuenta propia".

8. "Empleador o patrón, es el trabajador independiente que emplea los servicios de uno o varios trabajadores a cambio de una remuneración económica en monetario o especie".

9. "Por cuenta propia, es el ocupado que trabaja solo o con el apoyo de integrantes de su propio hogar o ajenos, pero sin el compromiso de pagarles por sus servicios".

10. "Persona ocupada que no recibe ningún tipo de pago (monetario o en especie), por su ocupación. Aunque es susceptible de recibir algún tipo de prestaciones".
Por su parte, los empleadores o patrones, también en 2014, superan en 0.7 puntos porcentuales la participación que obtuvieron en 2013 (5.7 por cada 100 trabajadores son patrones), la que a su vez, resulta superior en 1.5 puntos porcentuales la participación que éstos registran a escala nacional. En contraste, para 2014, se encuentra la participación de los trabajadores por cuenta propia y la de los no remunerados. Ambos registran una contracción de 2.4 y 0.3 puntos porcentuales, respectivamente (Gráfico 3).

Sin embargo, en 2014, 70 mil 719 personas que formaban parte de la población ocupada experimentan una pérdida de su empleo (4 por ciento con respecto al total de población ocupada en 2014). De acuerdo a la información estadística, la pérdida de empleo difiere entre cada uno de los segmentos de trabajadores que configuran la estructura ocupacional de la RMG. El segmento de trabajadores por cuenta propia reporta una pérdida de 38 mil 259 empleos (54.1 por ciento con respecto al total de personas que perdieron su ocupación en 2014); el de los trabajadores subordinados y remunerados pierden 26 mil 095 empleos (36.9 por ciento con respecto al total de pérdidas); y, el de no remunerados pierden 6 mil

Gráfico 3. RMG: participación porcentual de los trabajadores por tipo de ocupación, 2007-2014

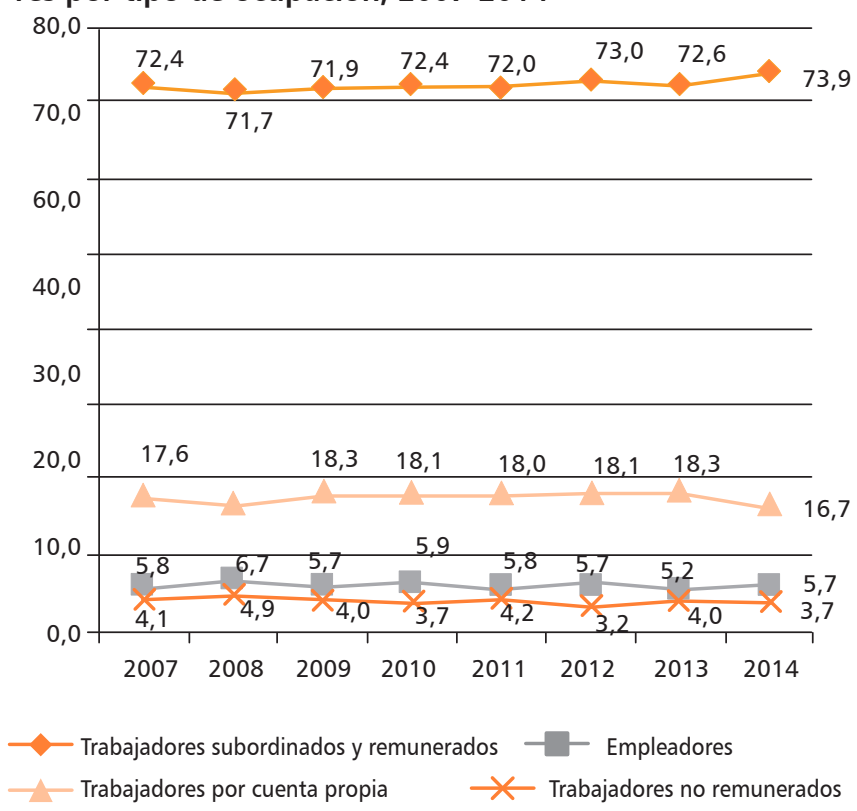

Fuente: Elaboración propia con base en INEGI, Encuesta Nacional de Ocupación y Empleo (ENOE). 
365 empleos (9 por ciento del total de pérdidas). Estas pérdidas no lograron ser compensadas por el incremento que experimentaron los empleadores o patrones (4 mil 634). Las pérdida de empleo que experimenta cada uno de los tres segmentos de trabajadores mencionados, así como, el incremento del número de patrones, es la consecuencia, por una parte, de aspectos estructurales (ritmo del proceso de acumulación: mayor productividad laboral en algunas ramas de actividad principalmente de la industria manufacturera; mayor inversión extranjera; menor capital variable; bajo ritmo de comercialización de bienes y servicios en el mercado interno, entre otros), por otra, de aspectos institucionales (como modificación, en 2012, de la ley federal del trabajo ${ }^{11} \mathrm{y}$, del régimen fiscal).

Otro matiz en la pérdida de empleo, lo proporciona, la tasa de crecimiento anual que experimenta cada segmento de trabajadores. Como se observa en la gráfica 4, para 2014, los trabajadores por cuenta propia experimentan la mayor tasa de crecimiento negativa (12.2 por ciento), la que contrasta con la tasa de crecimiento positiva registrada durante el periodo 2009-2013: entre 2.0 y 7.3 por ciento. Cabe señalar que, en 2009 , solo este segmento de trabajadores registra un crecimiento de 7.3 por ciento. Se puede inferir que el trabajo por cuenta propia, representa una estrategia para enfrentar el mayor desempleo que generan las crisis económicas y el mayor ritmo de acumulación de capital, pero no, para enfrentar los cambios de régimen fiscal como ocurrió el año pasado.

Por su parte los trabajadores no remunerados, también experimentan, en 2014, un crecimiento anual negativo de 9.4 por ciento. Cabe señalar que, durante el periodo analizado este segmento de trabajadores registra las mayores tasas de crecimiento y decrecimiento en su nivel de ocupación respecto al resto de segmentos de trabajadores. Su dinámica, con excepción de 2008, es inversamente proporcional a la ostentada por los restantes tres segmentos de trabajadores.

11. En los cambios de la ley federal del trabajo, destacan la flexibilización de la compra-venta de la fuerza de trabajo a través de la ampliación de los contratos a prueba y por periodo de aprendizaje; de la legalización de las agencias de colocación; del pago por hora y del abaratamiento de la indemnización por con concepto de despido y/o liquidación por cualquier causa que lo provoque.
En tercer término se encuentra la tasa de crecimiento negativa de la fuerza de trabajo o trabajadores subordinados y remunerados (-2.1 por ciento). A partir de 2012, sostiene una dinámica descendente y también un comportamiento en su dinámica muy parecido al segmento de trabajadores por cuenta propia. Cabe señalar que su comportamiento está ligado de manera directa al ritmo del proceso de acumulación de capital que impera en la industria manufacturera de la RMG en la medida que compran y despiden fuerza de trabajo en razón de los requerimientos de dicho proceso.

Mención especial merece la dinámica del segmento de patrones. En 2014 registra un crecimiento positivo de 4.9 por ciento inferior al de 2008 (21.5 por ciento) y 2010 (6.7 por ciento) pero superior al resto de los años del periodo analizado (véase gráfico 4). Cabe señalar que el crecimiento del año pasado se asienta en buena medida en el incremento de los patrones del sector agropecuario (4 mil 098 nuevos empleadores), el cual dicho sea de paso no es objeto de análisis en el presente trabajo, incremento que a su vez se relaciona de manera directa con el cambio de régimen tributario que se gestó en 2014. Gráfico 4. RMG: tasa de crecimiento anual de los trabajado-
res según posición en la ocupación, 2008-2014

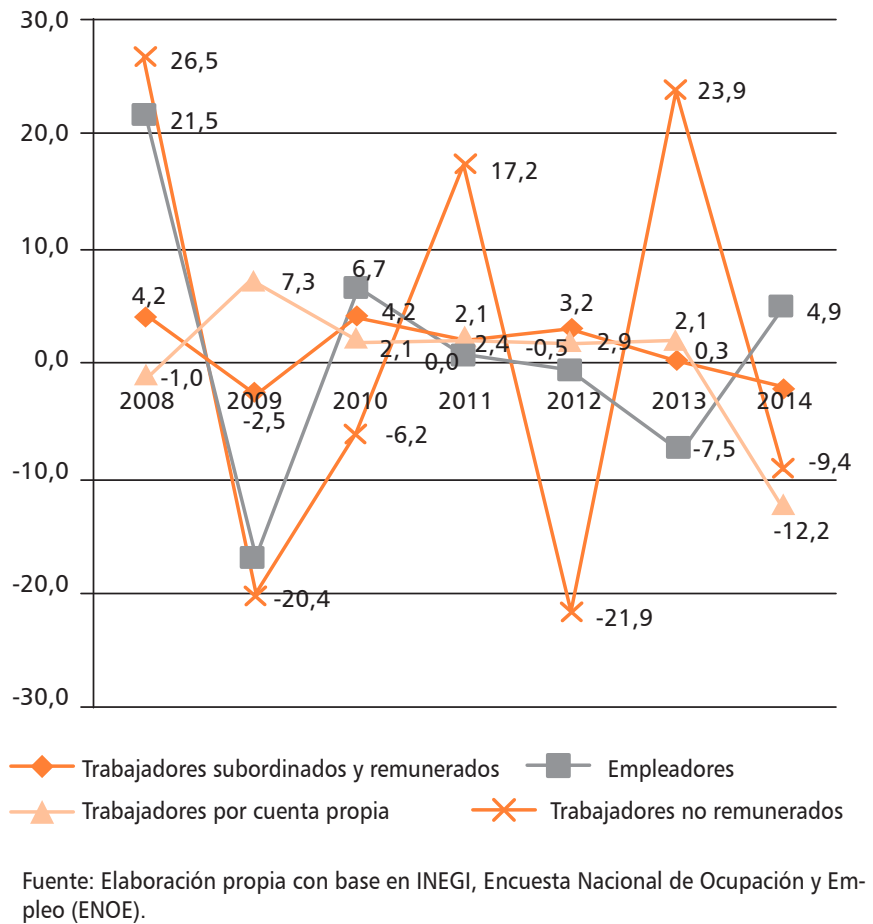
pleo (ENOE). 


\subsubsection{Nivel de ocupación según espacio de actividad} económica

Las actividades económicas urbanas son: industria de la construcción, industria manufacturera, comercio (al mayoreo y menudeo) y servicios. Del universo de trabajadores ocupados en la RMG, en 2014, solamente los de la industria de la construcción registraron un crecimiento en términos absolutos (14 mil 943), así como en términos relativos (12.8 por ciento). En contraste, las mayores pérdidas de empleo las experimentaron los trabajadores de la industria manufacturera (-9.4 por ciento), del comercio ( -4.3 por ciento) y, de los servicios con -4.3 por ciento. La suma total de pérdidas de empleo por parte de estas tres industrias es de 92, 916 personas, cantidad que disminuye, como se mencionó anteriormente a 70,719 personas, por la compensación que produce la creación de empleos por parte, principalmente, de la industria de la construcción y, del sector agropecuario.

Al correlacionar actividad económica con segmento de trabajadores, los resultados son los siguientes. La generación de empleo en la industria de la construcción permitió que los trabajadores: subordinados y remunerados registraran un crecimiento de 14 por ciento; por cuenta propia, 15.3 por ciento; no remunerados, 36 por ciento. Solamente los empleadores, experimentan una pérdida de 8.8 por ciento en su participación en dicha industria.

Por otra parte, los empleadores o patrones, obtienen un crecimiento importante en la industria manufacturera (10.4 por ciento) y, en los servicios, de solo 1.1 por ciento lo cual significa generación de empleos. En contraste, los trabajadores no remunerados, experimentan en la industria manufacturera, la mayor pérdida de empleos con una tasa de crecimiento anual de 16.1 por ciento. En esta misma industria, los trabajadores: por cuenta propia registran un crecimiento negativo de 15.6 por ciento y, los subordinados y remunerados de 10 por ciento.

En la actividad de servicios el mayor retroceso ocupacional, también corresponde a los trabajadores no remunerados con 23.9 por ciento (este segmento de tra- bajadores registra la mayor dinámica negativa y positiva en el conjunto de la actividad económica de la RMG). Por su parte los trabajadores por cuenta propia, también alcanzan una pérdida de 17.1 por ciento y por último, los trabajadores subordinados y remunerados una dinámica negativa de 1.1 por ciento.

Mención especial merece la actividad comercial, toda vez que los cuatro segmentos de trabajadores obtuvieron pérdidas en la ocupación. La mayor pérdida corresponde a los trabajadores por cuenta propia con una tasa de 10.9 por ciento; remunerados y subordinados, -1.9 por ciento; empleadores y no remunerados, ambos, 0.4 por ciento (Gráfico 5).

Las pérdidas de empleo, por sí mismas sugieren que con un menor número de trabajadores ocupados, se produjo una mayor riqueza como producto de la ampliación e intensificación del proceso de acumulación de capital. La acumulación presupone un incremento, por ejemplo, en la compra de materias primas y en la fuerza de trabajo. Estas compras se observan en las empresas de la industria manufacturera, maquiladora y de servicios de exportación (IMMEX). Las empresas IMMEX localizadas en los municipios de Guadalajara y Zapopan, representan el

Gráfico 5. RMG: tasa de crecimiento anual de los trabajadores ocupados según actividad económica, 2014

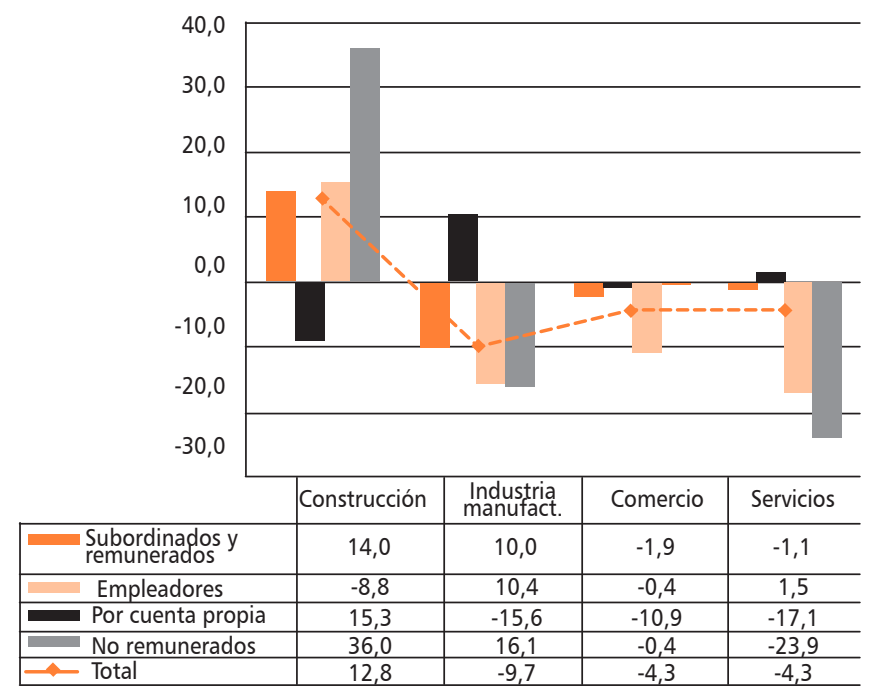

Fuente: Elaboración propia con base en INEGI, Encuesta Nacional de Ocupación y Empleo (ENOE). 
60 por ciento del número total de dichas empresas establecidas en Jalisco, registran un incremento de materias primas nacionales, en 2014, del 18.9 y 2.7 por ciento, respectivamente. Asimismo, estas empresas pagaron un mayor monto de remuneraciones a su fuerza de trabajo en Guadalajara: 0.2 por ciento y, en Zapopan: 44.2 por ciento (INEGI, 2015a). Por su parte la generación de mayor riqueza, presupone el incremento de la productividad laboral en dichas plantas manufactureras mediante una mayor intensificación del trabajo, mayor inversión extranjera destinada a la apertura de nuevas empresas así como la destinada a la adquisición de tecnología, entre otros aspectos.

\subsection{Nivel de ingresos}

En este apartado se analiza el nivel de ingresos con el fin de ofrecer evidencia, principalmente, sobre la pérdida del poder adquisitivo. Por una parte, se destaca la pérdida del poder adquisitivo del salario mínimo con respecto a la canasta alimenticia recomendable (CAR) ${ }^{12}$. Por otra, la pérdida del poder adquisitivo del salario que percibe cada segmento de la población que cuenta con un empleo respecto a la CAR. Ello, con el fin de matizar el deterioro que enfrentan los trabajadores ocupados según su posición en la estructura ocupacional. Sobre la base de dicha evidencia empírica, se infiere que se produce un incremento en la precariedad laboral de los trabajadores ocupados en la RMG.

\section{- Poder Adquisitivo del Salario Mínimo en México}

El ingreso monetario, constituye una variable de primer orden para el proceso de reproducción de los trabajadores y de sus familias, así como, para el proceso de producción y reproducción del capital. Con respecto al primero, destacan las remuneraciones al trabajo. En México, las remuneraciones al trabajo han experimentado, desde 1995, un consistente deterioro: de un 35 por ciento de participación en el PIB en 1994, a un 27 por ciento en 2013 (CEPAL, 2014). Este consistente deterioro macroeconómico desde la perspectiva marxista "equivale a una elevación de la tasa de explotación (Husson, 2009).

Por otra parte destaca el salario mínimo (SM). El SM nominal de 2014, respecto al de 1987 ha experimentado un incremento de 940 por ciento. En contraste, el precio nominal diario de la CAR registra, para los mismos años, un incremento de 4,773 por ciento. Lo que significa que el precio de los alimentos registra un aumento de más de 4 a 1 con respecto al incremento de los SM. Más aún, con el monto del SM diario, en 2014, solo se puede adquirir poco más de un tercio de la CAR. Esto significa que el SM real acumula, entre 1987 y 2014, una pérdida en su poder adquisitivo de 78.7 por ciento (Cuadro 2).

Cuadro 2. Precio de la canasta alimenticia recomendable y poder adquisitivo del salario mínimo diario en México, 1987-2014

\begin{tabular}{|c|c|c|c|c|c|c|c|}
\hline Año & $\begin{array}{l}\text { Salario Mínimo } \\
\text { Diario (precio } \\
\text { nominal) }\end{array}$ & $\begin{array}{l}\text { Incremento } \\
\text { Porcentual del } \\
\text { Salario Mínimo } \\
\text { Nominal Diario }\end{array}$ & $\begin{array}{l}\text { Precio Diario de } \\
\text { la CAR (pesos } \\
\text { nominales) }\end{array}$ & $\begin{array}{l}\text { Incremento } \\
\text { Porcentual } \\
\text { Acumulado del } \\
\text { Precio de la CAR }\end{array}$ & $\begin{array}{l}\text { Porcentaje de } \\
\text { la CAR que se } \\
\text { puede adquirir } \\
\text { con } 1 \text { salario } \\
\text { mínimo }\end{array}$ & $\begin{array}{l}\text { Índice del } \\
\text { Salario Mínimo } \\
\text { Real }\end{array}$ & $\begin{array}{l}\text { Poder Adquisi- } \\
\text { tivo Acumulado }\end{array}$ \\
\hline 1987 & 6.47 & 0.0 & 4.0 & 0.0 & 163.8 & 100.0 & 0.0 \\
\hline 2006 & 48.67 & 652.2 & 80.8 & 1946.3 & 60.2 & 36.8 & -63.3 \\
\hline 2014 & 67.29 & 940.0 & 192.5 & 4773.0 & 35.0 & 21.3 & -78.7 \\
\hline
\end{tabular}

Fuente: Tomado de CAM-UNAM, 2014, p. 2

12. La CAR, incluye 38 alimentos cuyos nutrientes son los mínimos necesarios para una familia de 3.5 miembros. Esta canasta fue elaborada por el Instituto Nacional de Nutrición Salvador Zubirán de la Secretaría de Salud y el Centro de Análisis Multidisciplinario de la UNAM. Con base en los cálculos realizados por este Centro de Análisis, establece que para que un trabajador adquiera esta canasta, sin considerar gastos en vivienda, salud, transporte, educación, etc., debe percibir en promedio 3 salarios mínimos (Lozano et al, 2012). 


\subsubsection{Nivel salarial porsegmento de trabajadores}

En este sub-apartado se destacan los niveles salariales ${ }^{13}$ que percibe cada segmento de trabajadores, los que a su vez, se contrastan con el precio de la canasta alimenticia recomendable (CAR). Ello, con el fin de establecer el número de trabajadores que, en 2014, perciben el nivel salarial suficiente para adquirir la canasta alimenticia recomendable para una familia de 3.5 miembros. De acuerdo con lo anterior, se configuran tres grupos de trabajadores: uno, los de nivel muy bajo-bajo: perciben entre 1 y 3 salarios mínimos insuficiente para obtener la CAR; dos, los de nivel medio: perciben más de 3 y hasta 5 salarios mínimos; los medio-altos: perciben más de 5 salarios mínimos. Los dos últimos grupos si obtienen los recursos económicos para comprar la referida canasta alimentaria recomendable.
En 2014, el 6.8 por ciento del total de trabajadores ocupados $(118,971)$ obtuvo un ingreso de hasta un salario mínimo. Este nivel salarial lo perciben tres de los segmentos de trabajadores: 5.2 por ciento del total de trabajadores remunerados y subordinados; 1.4 por ciento del total de empleadores y, 17.1 por ciento del total de trabajadores por su cuenta (Cuadro 3).

Asimismo, del total de trabajadores que perciben hasta un salario mínimo, el 51 por ciento labora en la rama de servicios; 36 por ciento en el comercio y 10 por ciento en la industria manufacturera.

Por otra parte, destaca el 21.2 por ciento del total de la población ocupada que percibe más de uno y hasta 2 salarios mínimos: 1.3 puntos porcentuales inferior al registrado en 2013. Del total de trabajadores subordinados y remunerados, 22.9 por ciento percibe más de 1 y

Cuadro 3. RMG: Participación porcentual de los trabajadores por segmento según nivel salarial**

\begin{tabular}{|c|c|c|c|c|c|c|}
\hline Posición en la Ocupación & $\begin{array}{l}\text { Hasta un salario } \\
\text { mínimo }\end{array}$ & $\begin{array}{l}\text { Más de } 1 \text { hasta } \\
2 \text { salarios míni- } \\
\text { mos }\end{array}$ & $\begin{array}{l}\text { Más de } 2 \text { hasta } \\
3 \text { salarios míni- } \\
\text { mos }\end{array}$ & $\begin{array}{l}\text { Más de } 3 \text { hasta } \\
5 \text { salarios míni- } \\
\text { mos }\end{array}$ & $\begin{array}{l}\text { Más de } 5 \text { sala- } \\
\text { rios mínimos }\end{array}$ & $\begin{array}{l}\text { No recibe } \\
\text { ingresos }\end{array}$ \\
\hline \multicolumn{7}{|l|}{2014} \\
\hline Total & 6.8 & 21.2 & 30.1 & 22.7 & 9.2 & 3.7 \\
\hline $\begin{array}{l}\text { Trabajadores subordinados y } \\
\text { remunerados }\end{array}$ & 5.2 & 22.9 & 34.7 & 22.9 & 8.7 & \\
\hline Empleadores & 1.4 & 8.6 & 15.9 & 34.5 & 27.3 & \\
\hline Trabajadores por cuenta propia & 17.1 & 22.9 & 21.3 & 22.9 & 6.8 & \\
\hline \multicolumn{7}{|l|}{2013} \\
\hline Total & 6.8 & 22.5 & 26.1 & 21.7 & 10.2 & 4.0 \\
\hline $\begin{array}{l}\text { Trabajadores subordinados y } \\
\text { remunerados }\end{array}$ & 4.8 & 24.5 & 28.9 & 23.8 & 9.9 & \\
\hline Empleadores & 1.3 & 5.3 & 21.9 & 25.2 & 28.6 & \\
\hline Trabajadores por cuenta propia & 17.9 & 24.5 & 21.5 & 17.4 & 8.5 & 0.1 \\
\hline
\end{tabular}

** Sólo el 93 por ciento de trabajadores encuestados especificó su nivel salarial.

Fuente: Elaboración propia con base en INEGI, Encuesta Nacional de Ocupación y Empleo (ENOE).

13. En México los salarios mínimos (SM) son determinados por una institución gubernamental: Comisión Nacional de Salarios Mínimos la cual es responsable de la contención salarial. En esta Comisión, participa el sector privado, las principales centrales de trabajadores y actores públicos que con base en la estimación de la inflación cada primero de enero, determinan el incremento del SM del año en curso. Asimismo, en la contención salarial incide la gran informalidad del mercado laboral en la RMG, el 44 por ciento del empleo total, en 2014, es informal y, también la falta de un seguro de desempleo, así como el ejército de reserva de fuerza de trabajo que lo configuran, principalmente, los desempleados, así como la población dispuesta a trabajar pero que no busca empleo. 
hasta 2 salarios mínimos; del total de empleadores el 8.6 por ciento percibe dicho ingreso y; el 22.9 por ciento del total de trabajadores por su cuenta perciben esta misma cantidad (Cuadro 3).

Por su parte los trabajadores que obtienen más de 2 y hasta 3 salarios mínimos, representan casi un tercio del total de trabajadores ocupados en 2014: incremento de 4 puntos porcentuales respecto a la participación registrada un año antes. Del número total de subordinados y remunerados, 34.7 por ciento perciben dicho nivel salarial; del total de empleadores, 15.9 por ciento devengan este mismo nivel salarial y, 21.3 por ciento de los trabajadores por su cuenta (Cuadro 3). De este conjunto de trabajadores el 44 por ciento labora en los servicios; 23 por ciento en el comercio y otro 23 por ciento en la industria manufacturera; $y$, el 10 por ciento en la industria de la construcción, (INEGI, 2015a).

En suma, del total de trabajadores con menos de 1 hasta 3 salarios mínimos, 48 por ciento labora en la rama de servicios; 26 por ciento en la rama de comercio y, 19 por ciento en la industria manufacturera.

En cuarto término, destaca el 22.7 por ciento de los trabajadores que perciben más de 3 y hasta 5 salarios mínimos. Del total de subordinados y remunerados, el 22.9 por ciento percibe este nivel salarial; del total de empleadores, el 34.5 por ciento percibe este mismo nivel salarial y, el 17.4 por ciento también percibe esta cantidad (véase cuadro 2). Su ocupación se distribuye de la manera siguiente: servicios, 55 por ciento; comercio, 18 por ciento; industria manufacturera, 19 por ciento (INEGI, 2015a).

Por último, se encuentra el 9.2 por ciento de trabajadores que perciben más de 5 salarios mínimos, ocupados en las mismas ramas de actividad y en las mismas proporciones que los trabajadores del anterior nivel salarial. La mayor proporción corresponde a los empleadores: 27.3 por ciento; los subordinados y remunerados: 8.7 por ciento y, por cuenta propia: 6.8 por ciento (véase cuadro 3 ).

De acuerdo a todo lo anterior, el 58.1 por ciento del total de trabajadores ocupados en la RMG percibe entre menos de 1 hasta 3 salarios mínimos: 2.7 puntos porcentuales superior a la proporción registrada en 2013.
Esto significa que al cabo de un año, 2.7 por cada 10 trabajadores ocupados se sumaron a este nivel salarial. Por lo tanto, en 2014, 5.8 de cada 10 trabajadores no perciben, en promedio, el nivel salarial suficiente para comprar la CAR. En consecuencia, esta desvalorización salarial contribuye de manera importante a ampliar y profundizar las condiciones de precariedad laboral en la RMG, así como a incrementar la desigualdad salarial que contribuye de manera sustantiva al empobrecimiento de un mayor número de población.

En contraste, se observa la participación de los trabajadores que perciben más de 3 y hasta 5 salarios mínimos 22.7 por ciento (un punto porcentual superior al registrado en 2013). Sin embargo, dicho punto porcentual, se supone, corresponde al mismo que pierden los trabajadores que perciben más de 5 salarios (Cuadro 3). Por lo anterior se infiere que el 1 por ciento, de este último segmento de trabajadores continúa laborando pero percibiendo un menor ingreso. Todo ello, constituye otra evidencia sobre el nivel de deterioro salarial que alcanza este conjunto de trabajadores que laboran en la RMG. No obstante lo anterior, se trata de casi un tercio de trabajadores, misma proporción que la registrada en 2013, que por el nivel salarial que perciben se encuentran en condiciones de poder adquirir la CAR. Sin embargo, los de hasta 5 salarios mínimos, nivel medio, se encuentran en desventaja frente a los del nivel medio-alto (más de 5 salarios mínimos) respecto al consumo de otros bienes y servicios.

Esta contradicción entre aumento en el deterioro de las condiciones laborales y de vida para la mayoría de trabajadores ocupados y el estancamiento de un tercio de trabajadores que solventan sus necesidades mínimas y algo más, son el resultado del ritmo que impone el proceso de acumulación de capital en consonancia con las modificaciones a la ley laboral y al régimen fiscal vigente.

Otro matiz sobre el nivel salarial, corresponde a la participación porcentual que registra cada segmento de trabajador ocupado. El 62.8 por ciento del total de trabajadores subordinados y remunerados, perciben entre $1 \mathrm{y}$ 3 salarios mínimos, 4.6 puntos porcentuales más que los 
registrados en 2013. Lo cual significa que para una mayor cantidad de fuerza de trabajo ocupada en la RMG, le resulta inaccesible la adquisición de la canasta alimenticia recomendable. En contraste, otro 22.9 por ciento del total de la misma fuerza de trabajo ocupada percibe más de 3 y hasta 5 salarios mínimos: un punto porcentual mayor a la proporción de 2013. Otro 9.2 por ciento del total de la fuerza de trabajo percibe más de 5 salarios mínimos. En suma, solamente una cuarta parte del total de la fuerza de trabajo se encuentra en condiciones de adquirir la CAR y algo más de bienes y servicios.

En segundo término se ubican los empleadores. Por cada 100 patrones, 26 perciben entre 1 y 3 salarios mínimos. Ingreso insuficiente para adquirir la canasta alimenticia. Se trata de un conjunto de patrones que impulsan actividades de bajo nivel agregado, además que se ubican, principalmente, en micro establecimientos. En contraste, 34.5 por cada 100 empleadores perciben un ingreso de más de 3 y hasta 5 salarios mínimos. Cabe señalar que, 9.3 empleadores se sumaron, en 2014, a este nivel de ingresos. Por último, destaca el 27.3 por ciento de los empleadores que perciben más de 5 salarios mínimos. De acuerdo a lo anterior, 43.8 por cada 100 empleadores perciben ingresos suficientes para adquirir la susodicha canasta alimenticia. No obstante lo anterior, 10 empleadores menos dejaron de percibir dicho nivel de ingresos, toda vez que en 2013 sumaban 53.8. En tercer término destacan los trabajadores por cuenta propia. Por cada 100 trabajadores por cuenta propia 61.3 registran un nivel entre 1 y 3 salarios mínimos. Esto pone en evidencia que la actividad económica que desempeñan estos trabajadores, resulta escasamente calificada y poco remunerada sin contar con los recursos suficientes para adquirir la canasta alimenticia. En contraste, otro 22.9 por ciento de estos trabajadores percibe más de 3 y hasta 5 salarios mínimos. Esta participación representa un incremento de 5.5 puntos porcentuales respecto a 2013 .

Con base en la evidencia estadística ofrecida anteriormente, se matiza un persistente deterioro salarial que en amplitud (todos los segmentos de trabajadores y ramas de actividad económica enfrentan), profundidad (mayor número de trabajadores con un nivel salarial bajo) y dinamismo (véase gráfica 6) prevalece en el mercado de trabajo regional. Tan severo y dinámico resulta el deterioro salarial, que cada vez un mayor número de población ocupada (fuerza de trabajo, patrones, así como trabajadores por cuenta propia y no remunerados) no logran obtener los recursos económicos suficientes para adquirir la canasta alimenticia recomendable.

A su vez, de manera contradictoria también persiste un grupo importante de población ocupada que se ubica en un nivel salarial medio y medio-alto. Lo cual resulta un indicador importante para observar cómo aumenta la población trabajadora con menores ingresos y disminuye la que obtiene ingresos salariales medios y altos. Asimismo, los magros ingresos salariales suscitan repercusiones negativas sobre el consumo privado el que a su vez impacta directamente en el PIB y en consecuencia sobre el conjunto de la actividad económica sobre todo del mercado nacional y regional. Es decir, la caída de los salarios en la RMG tiene distintas implicaciones que van desde el aumento a la pobreza hasta el estancamiento del mercado interno.

Gráfico 6. RMG: Tasa de crecimiento anual del nivel salarial según segmento de trabajadores, 2014

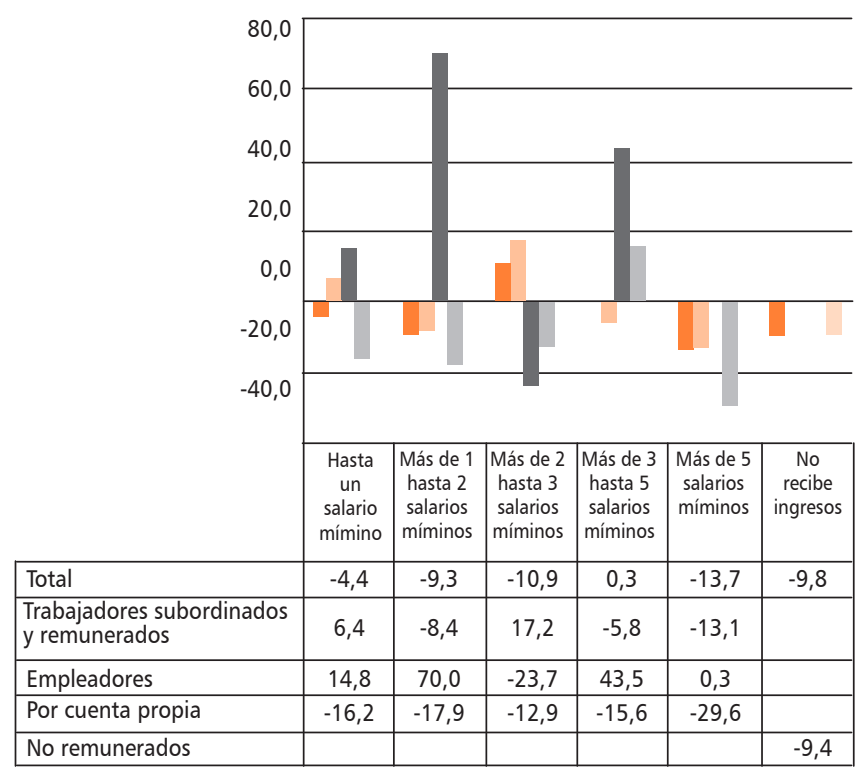

Fuente: Elaboración propia con base en INEGI, Encuesta Nacional de Ocupación y Empleo (ENOE). 


\section{Consideraciones finales}

En los postulados y el espíritu del artículo 123 constitucional de México, así como en los preceptos de toda ética de responsabilidad social se considera vital garantizar empleo para toda la población, así como una remuneración que permita al trabajador y a su familia reproducirse en condiciones decentes. Sin embargo, el Estado mexicano, conjunto de aparatos en sus tres niveles de gobierno, en santa alianza con el capital nacional y extranjero contravienen dichos postulados al impulsar, desde hace más de tres décadas, una serie de políticas que tienden a acentuar y agravar en extremo el deterioro laboral de todos los trabajadores en México. Como se puso en evidencia, la población trabajadora de la RMG enfrenta actualmente un deterioro laboral superior al que produjo la crisis económica a escala nacional, regional e internacional en 2009. Actualmente, se observa una mayor desigualdad laboral: Mayor pérdida de empleos versus Menor creación de empleo; Mayor número de trabajadores ocupados que perciben entre 1 y 3 SM vs. Menor número de trabajadores con ingresos de entre 3 y más de 5 salarios mínimos.

Este deterioro que observamos en ambas dimensiones laborales, contravienen los postulados constitucionales, así como, los preceptos de toda ética de responsabilidad social, y tienden a configurar una crisis laboral para el mayor número de trabajadores regionales. Observable, de acuerdo a la evidencia ofrecida, en distintos niveles y matices para el conjunto de trabajadores de acuerdo a su posición en la estructura ocupacional, así como en la rama de actividad económica donde laboral o dejaron de laborar..

En contraste, se observa, aunque exiguo, un crecimiento económico que experimenta la RMG; acompañado por un aumento tanto en la productividad laboral agregada, como en la inversión extranjera directa. Indicios de un incremento, en algunas ramas económicas, principalmente de la industria manufacturera, en el ritmo de acumulación de capital. A su vez, este comportamiento económico, impulsado por un número reducido de empresas manufactureras (grandes y medianas) orientadas al mercado externo en consonancia con los cambios en la LFT y el régimen fiscal, tiende a configurar la actual debacle laboral regional.

En suma, el análisis del nivel del empleo y de los niveles salariales de los trabajadores de la RMG, en sus diferentes matices y dimensiones, permite dimensionar la crisis laboral que la gran mayoría de éstos enfrentan al producirles un mayor deterioro en sus condiciones de trabajo y vida.

\section{Bibliografía}

- Aguiar, Santiago (2014). "La flexiprecarización Innovación, clase obrera y nueva precariedad: avance de una investigación”, Cuadernos de Estudios del Trabajo, núm.14, www.estudiosdeltrabajo.clac, consultado: 17 de mayo 2014

- Aguilar Adrián e Irma Escamilla (2000). "Reestructuración Económica y mercado laboral metropolitano", en Rocío Rosales Ortega (coordinadora), Globalización y regiones en México, UNAM-MA Porrúa.

- Antunes, Ricardo (2012). Prefacio del texto de Adrián Sotelo Valencia, Los rumbos del Trabajo. Superexplotación y precariedad social en el siglo XXI, MA Porrúa-UNAM

- Alves, Giovanni (2007). Dimens】es da reestrturação productiva. Ensaios de soiologís do trabalho, São Paulo, Editora Praxis.

- BANAMEX (2015). Indicadores Regionales de Actividad. Estimaciones deL PIB elaboradas por el Departamento de Estudios Económicos.

- Calva, José Luis y César Armando Salazar (2012). "Empleo digno y crecimiento económico" en José Luis Calva (coordinador), Empleo digno, distribución del ingreso y bienestar, Análisis Estratégico para el Desarrollo, Vol.11 Juan Pablos

- CAM-UNAM (2014). "El Salario Mínimo en México: de la pobreza a la miseria. Pérdida del 78.66\% del poder adquisitivo del salario", Reporte de investigación 117.

- CEPAL (2014). La participación del trabajo en el 
ingreso nacional. El regreso a un tema olvidado, Serie Estudios y Perspectivas número 157.

- García Guzmán, Brígida (2010). "Inestabilidad laboral en México: el caso de los contratos de trabajo". Estudios Demográficos y Urbanos, vol. 25, núm. 1, enero-abril, pp. 73-101. El Colegio de México, A.C. (2009)

- "Precariedad Laboral y Desempleo en México, 2000. 2009”. Ponencia presentada en la sesión especial "La participación económica en el contexto actual de crisis económica en América Latina", X Reunión Nacional de Investigación Demográfica en México, organizada por la Sociedad Mexicana de Demografía.

- Gaxiola Robles Linares, Sergio Cuauhtémoc (2013), "Análisis comparativo de la precariedad laboral en las tres principales metrópolis mexicanas para el 2010", Revista Facultad de Ciencias Económicas: Investigación y Reflexión, Vol. XXI (1), Universidad Militar Nueva Granada, Bogotá, Colombia, Junio, 131-146.

- Gómez Solórzano Marco Augusto (2007). "La precariedad siempre existente", en Mabel Burin, Marla Lucero Jiménez Guzmán, Irene Meler (compiladoras), Precariedad laboral y crisis de la masculinidad. Impacto sobre las relaciones de género. CUCES, Argentina, 2007

- Hualde y Guadarrama (2012). "Precariedad laboral y heterogeneidad ocupacional: una propuesta teórico- metodológica", Revista Mexicana de Sociología 74, núm. 2 (abril-junio, 2012)

- Husson, Michel (2009). Capitalismo Puro, Maia ediciones

- INEGI (2015). Encuesta Nacional de Ocupación y Empleo (ENOE), www.inegi.org.mx, consultado el 17 de febrero de 2015

INEGI (2015a). Estadística Integral del Programa de la Industria Manufacturera, Maquiladora y de Servicios de Exportación (IMMEX), www.inegi.org. $\underline{\mathrm{mx}}$.

- Lozano Arredondo, Luis, et al., (2012). "Deterioro del salario bajo el neoliberalismo y necesidad de restaurar el valor de la fuerza de trabajo" en José Luis Calva (coordinador), Empleo digno, distribución del ingreso y bienestar, Análisis Estratégico para el Desarrollo, Vol.11 Juan Pablos.

- Parra Ruiz, José María y Emilia Gámez F. (2006). "Movilidad espacial de los trabajadores que laboran en la industria electrónica de la región metropolitana Guadalajara" Carta Económica Regional, núm. 91, Universidad de Guadalajara, enero-marzo, pp. 51-69, Guadalajara, Jalisco, México.

- Sotelo Valencia, Adrián (2012). Los rumbos del Trabajo. Superexplotación y precariedad social en el siglo XXI, M.A. Porrúa-UNAM. 